Research Paper

\title{
Biatrial enlargement as a predictor for reablation of atrial fibrillation
}

\author{
Qiang Kong리, Lisheng Shi ${ }^{1}$, Ronghui $\mathrm{Yu}^{2}$, Deyong Long${ }^{2}$, Yucong Zhang ${ }^{1}$, Yujia Chen ${ }^{1}$, Jing Li ${ }^{1 凶}$ \\ 1. Division of cardiology, Capital Medical University Xuanwu Hospital, No. 45 Changchun Street, Xicheng District, Beijing 100053, PR China \\ 2. Division of cardiology, Beijing Anzhen Hospital, Capital Medical University, No. 2 Anzhen Road, Chaoyang District, Beijing 100029, PR China. \\ \Corresponding author: E-mail: shpxbb@sina.com
}

(c) The author(s). This is an open access article distributed under the terms of the Creative Commons Attribution License (https://creativecommons.org/licenses/by/4.0/). See http://ivyspring.com/terms for full terms and conditions.

Received: 2020.04.29; Accepted: 2020.09.27; Published: 2020.10.18

\begin{abstract}
Purpose: We aimed to determine whether biatrial enlargement could predict reablation of atrial fibrillation after first ablation.

Methods: 519 consecutive patients with drug resistant atrial fibrillation [paroxysmal AF (PAF) 361, non-PAF 158] who underwent catheter ablation in Capital Medical University Xuanwu hospital between 2009 and 2014 were enrolled. Biatrial enlargement (BAE) was diagnosed according to trans-thoracic echocardiography (TTE). Ablation strategies included complete pulmonary vein isolation (PVI) in all patients and additional linear ablation across mitral isthmus, left atrium roof, left atrium bottom and tricuspid isthmus, or electrical cardioversion on the cases that AF could not be terminated by PVI. Anti-arrhythmic drugs or cardioversion were used to control the recurred atrial arrhythmia in patients with recurrence of atrial fibrillation after ablation. Reablation was advised when the drugs were resistant or that patient could not tolerate. Risk factors for reablation were analyzed.
\end{abstract}

Results: After $33.11 \pm 21.45$ months, 170 patients recurred atrial arrhythmia, and reablation were applied in 117 patients. Multivariate Cox regression analysis demonstrated that that biatrial enlargement (BAE, HR 1.755, $95 \% \mathrm{Cl} 1.153-2.670, \mathrm{P}=0.009)$ was an independent predictor for reablation and was associated with reablation (Log rank $\mathrm{P}=0.007$ ).

Conclusion: Biatrial enlargement is an independent risk predictor for the reablation in atrial fibrillation patients after first ablation.

Key words: Atrial fibrillation, Catheter ablation, Biatrial enlargement

\section{Introduction}

Atrial fibrillation (AF) is the most common sustained cardiac arrhythmia (1). Catheter ablation of pulmonary veins or left atrium have been proved to be superior to anti-arrhythmic therapy in reducing $\mathrm{AF}$ recurrence and maintaining sinus rhythm $(3,4)$. However, pulmonary vein isolation (PVI) or PVI plus additional linear ablation is associated with a considerable recurrence rate (5). Despite that anti-arrhythmic drugs or cardioversion were used to control the recurred atrial arrhythmia, almost $10 \%$ patients had to accept reablation due to atrial arrhythmias that refractory to anti-arrhythmic drugs (6). Risk factors have been identified to predict the recurrence of atrial fibrillation after ablation, such as left atrial enlargement (LAE), right atrium diameter (RAD), atrial tissue fibrosis, and low-voltage areas detected by high-density electroanatomical mapping in left atrium $(7,8,9,10,11)$. However, risk factors to predict the reablation in patients with recurrence of atrial fibrillation are still uncertain. Recent study (12) had found that biatrial volume were independent predictors of AF recurrence after PVI. However, the predictive value of atrial enlargement of reablation in patients with recurrence of $\mathrm{AF}$ is still unknown. In this study, we assessed biatrial enlargement as a predictor for reablation in atrial fibrillation patients.

\section{Methods}

\section{Study population}

All procedures were in accordance with the ethical standards of the responsible committee on 
human experimentation and with the Helsinki Declaration of 1964, which was revised in 2013. All experimental protocols were approved by the Institutional Review Boards of the Capital Medical University Xuanwu hospital. All participants provided written informed consent.

This was a retrospective study. We reviewed 519 patients undergoing their primary catheter ablation of symptomatic drug resistant AF (paroxysmal AF $\mathrm{n}=361$; non-paroxysmal AF $\mathrm{n}=158$ ) in the heart center of Capital Medical university Xuanwu hospital from January 2009 to December 2014. Patients with chronic pulmonary disease, pulmonary hypertension, right-sided heart disease such as Ebstein's anomaly, and arrhythmogenic right ventricular cardiomyopathy (ARVC) were excluded. Informed consent for the AF ablation procedure was obtained from patients.

\section{Echocardiography}

All the patients underwent trans-thoracic echocardiography (TTE) and trans-esophageal echocardiography (TEE) (Vivid, GE). The Left and right diameter was measured on the four-chamber apical view during systolic phase. The horizontal diameter of left atrium (LA) was determined as the measurement from the middle of mitral isthmus to the LA roof (endocardial surface), while the horizontal diameter of right atrium (RA) was determined as the measurement from the middle of tricuspid isthmus to the RA roof (endocardial surface), see Figure 1. LA and left atrial appendage (LAA) thrombus was excluded by TEE. Our standard techniques for echocardiography examination also included that all measurements were averaged from at least three cardiac cycles and then reviewed by two echocardiologists. Right atrium enlargement (RAE) was diagnosed from that the horizontal diameter of right atrium was above $40 \mathrm{~mm}$ regardless of sex. Left atrium enlargement (LAE) was diagnosed that left atrium horizontal diameter was above $40 \mathrm{~mm}$ for male and $35 \mathrm{~mm}$ for female. Biatrial enlargement (BAE) was diagnosed when a patient was with both LAE and RAE. The normal value of echocardiography measurements in the Chinese population referred to the consensus statements endorsed by the Beijing Task Force on Echocardiography (13).

\section{Electrophysiology study and AF radiofrequency ablation}

Antiarrhythmic drugs (AADs) were discontinued for at least five half-lives before ablation. Oral anticoagulation (warfarin) was discontinued 3 days prior to the procedure, and low molecular weight heparin was administered for bridging. The procedure was performed with patients under conscious sedation. First, a decapolar catheter was positioned in the coronary sinus through left subclavian vein. Then transseptal puncture was accomplished with Sanjude transseptal puncture

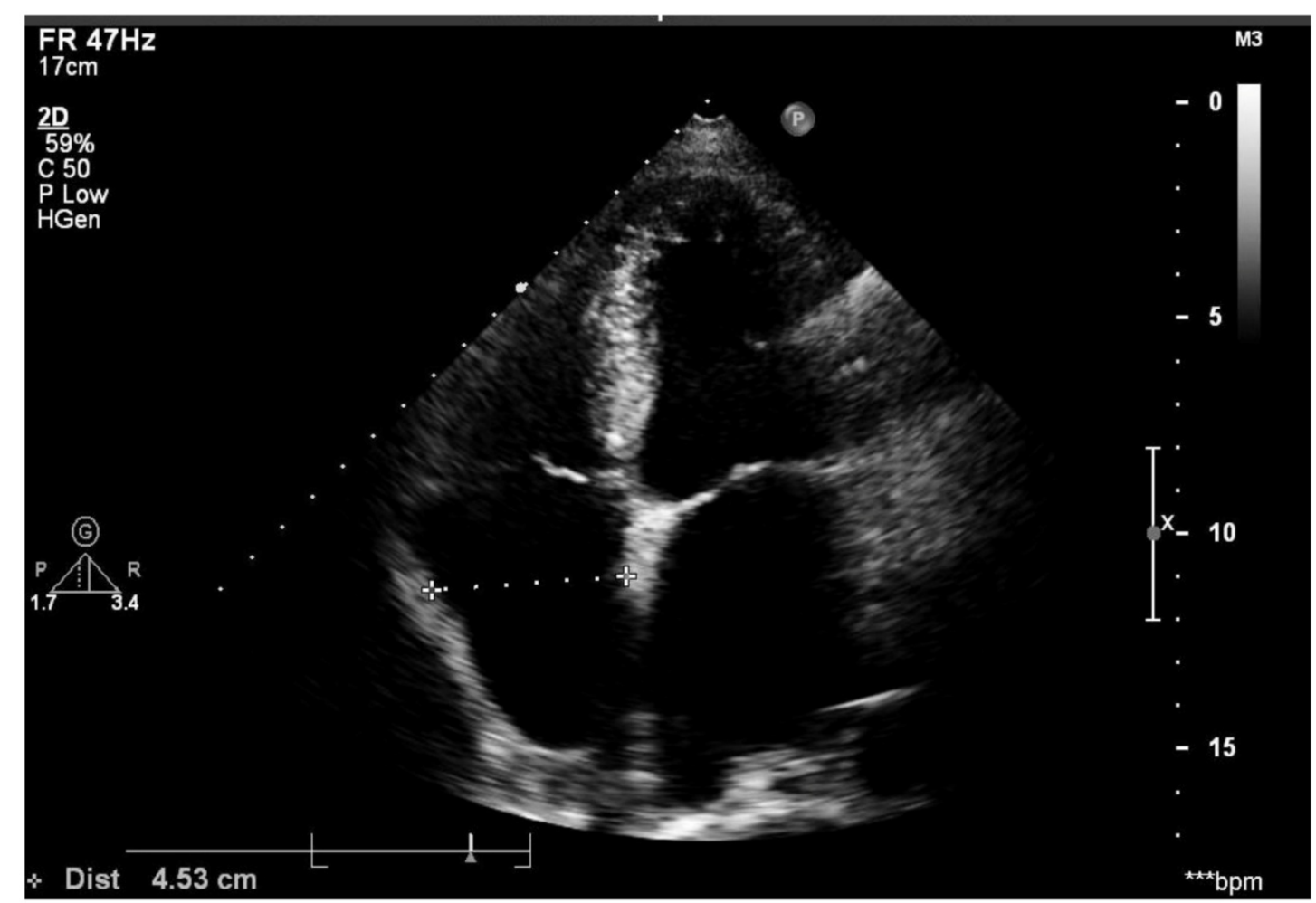

Figure 1. Right atrium diameter was measured using the four-chamber apical view during the systolic phase and left atrium diameter was measured also on this image. 
needle (St. Jude Medical Inc.). Following completion of transseptal access, a bolus of unfractionated heparin is given ( $50 \mathrm{U} / \mathrm{kg}$ body weight) and repeated for procedures lasting longer than $4 \mathrm{~h}$ or if the ACT falls below $200 \mathrm{~s}$. After that, a 3.5- $\mathrm{mm}$ irrigated ablation catheter (Biosense-Webster Inc, Diamond Bar, CA, USA) was advanced to the LA for mapping and ablation. Mapping and ablation were guided by a 3D electro-anatomical mapping system (CARTO, Biosense-Webster Inc.) or non-contact mapping system (Ensite NavX system, St. Jude Medical Inc). The sheath was continuously perfused with heparinized saline at $2-3 \mathrm{ml} / \mathrm{h}$.

Circumferential pulmonary vein isolation (PVI) was carried out for paroxysmal atrial fibrillation (PAF) patients. For persistent or long-standing persistent AF patients, additional linear ablation across LA roof, mitral isthmus, LA bottom and tricuspid isthmus were carried out besides PVI. Procedure endpoint was PVI for PAF patients and complete block of the lines besides PVI for persistent or long-standing persistent AF cases. Electrical cardioversion was applied if the atrial fibrillation could not terminated by PVI plus additional linear ablation. The specific definitions to PVI and linear block were detailed in previous report (14).

\section{Follow up}

After discharge, patients were followed by their referring cardiologist. During the first three postoperative months, anti-arrhythmic drugs such as amiodarone or propafenone or sotalol were used for every patient. Patients with AF recurrences which were drug resistant were advised to undergo electrical cardioversions within 3 months after ablation (blanking period). A 12-lead ECG was obtained at 3, 6, and 12 months in all patients. Patients with no recurrence and a CHADS-VASC score <2 stopped taking warfarin 3 months following the procedure. Anti-arrhythmic drug was stopped 3 months after ablation if a patient had no recurrence of atrial tachyarrhythmia. Recurrence of AF was defined as $\mathrm{AF}$ or atrial tachycardia lasting $>30$ seconds after blanking period. Anti-arrhythmic drugs were used for recurrence of atrial arrhythmia after blanking period. Reablation was advised when the drugs were resistant or that patient could not tolerate. Anticoagulation strategy was strictly carried out according to CHADS-VASC grading system for each patient.

\section{Statistical analysis}

For baseline characteristics, the KolmogorovSmirnov test was used to test the normality of distribution. Continuous variables are shown as mean \pm standard deviation (SD) and compared using a two-tailed Student $t$ test, while medians (Q1, Q3) and Mann-Whitney $U$ tests were used for non-normally distributed variables. Categorical data are reported as counts and percentage (\%) and between-group comparisons were made using the Pearson Chi-square or Fisher exact test. Variables that were statistically significant in univariate regression models ( $P$ value $<0.1$ ) were included in multivariate binary logistic regression model using a "forward conditional" method to determine whether they remained significant after adjustment for potential confounders. Risk factors associated with reablation were determined by a Cox regression model. All tests were two sided and $\mathrm{P}$ values $<0.05$ were considered statistically significant. Analyses were performed by IBM SPSS 25 (SPSS, IBM, Armonk, NY, USA).

\section{Results}

\section{Demographic characteristics of study population}

As in the Table 1, Patients with PAF $(n=361)$ had smaller LA and RA diameter, smaller LVEDD and cardiac output, higher LVEF and less heart failure compared with non-PAF individuals $(n=158)$. However, age, sex, hypertension, diabetes mellitus, CHADS2 sore and CHADS-VASC score were similar.

Table 1. Characteristics of the study group

\begin{tabular}{lllll}
\hline Value & Total & PAF(n=361) & Non-PAF(n=158) & P \\
\hline Age, years & $65.39 \pm 10.65$ & $65.60 \pm 10.77$ & $64.91 \pm 10.39$ & 0.493 \\
Sex (female), n (\%) & $209(40.3)$ & $147(40.7)$ & $62(39.2)$ & 0.771 \\
Hypertension, $\mathrm{n}(\%)$ & $288(55.5)$ & $202(56.0)$ & $86(54.4)$ & 0.774 \\
Diabetes mellitus, $\mathrm{n}(\%)$ & $111(21.4)$ & $80(22.2)$ & $31(19.6)$ & 0.280 \\
Heart failure, n (\%) & $29(5.6)$ & $12(3.3)$ & $17(10.8)$ & 0.001 \\
CHADS2 & $1(0,2)$ & $1(0,2)$ & $1(0,2)$ & 0.659 \\
CHADS-VASC & $2(1,3)$ & $2(1,3)$ & $2(1,3)$ & 0.911 \\
Left atrium diameter, mm & $41(36,45)$ & $38(35,42)$ & $44(41,48)$ & 0.000 \\
Right atrium diameter, mm & $36(33,39)$ & $35(32,37)$ & $39(37,44)$ & 0.000 \\
LVEF, \% & $66(60,70)$ & $66(61,70.5)$ & $64.5(59,68)$ & 0.004 \\
LVIDD, mm & $50(47,54)$ & $50(47,53)$ & $52(47,55)$ & 0.042 \\
Cardiac output, (L/s) & $5.5(4.7,6.6)$ & $5.35(4.7,6.1)$ & $5.85(5,7.1)$ & 0.000 \\
Mitral E peek, (cm/s) & $88(70,107)$ & $80(67,102)$ & $101(85,117.5)$ & 0.000 \\
\hline LVEF: left ventricular ejection fraction. LVIDD: left ventricular internal diameter at \\
end-diastole
\end{tabular}

\section{The overall procedural outcome}

According to right atrium diameter and left atrium diameter, patients were divided into two groups, Biatrial enlargement group $(\mathrm{BAE}, \mathrm{n}=100)$ and non-Biatrial enlargement group (Non-BAE, $n=419$ ). The overall procedural outcome and clinical characters were listed in the Table 2. As in the Table 2, the procedure time was longer, and the proportion of persistent AF, PVI plus additional linear ablation, electrical cardioversion, recurrence and reablation were higher in BAE group. 
Table 2. Clinical characters between BAE and Non-BAE

\begin{tabular}{|c|c|c|c|c|}
\hline Value & Total & $\operatorname{BAE}(n=100)$ & Non-BAE $(n=419)$ & $\mathrm{P}$ \\
\hline Age, years & $65.39 \pm 10.65$ & $65.39 \pm 10.38$ & $65.39 \pm 10.72$ & 0.944 \\
\hline Sex (female), n (\%) & $209(40.3)$ & $34(34.0)$ & $175(41.8)$ & 0.174 \\
\hline Hypertension, $\mathrm{n}(\%)$ & $288(55.5)$ & $56(56.0)$ & $232(55.4)$ & 0.909 \\
\hline Diebetes mellitus, $\mathrm{n}(\%)$ & $111(21.4)$ & $22(22.0)$ & $89(21.2)$ & 0.892 \\
\hline $\mathrm{CAD}, \mathrm{n}(\%)$ & $86(16.6)$ & $22(22.0)$ & $64(15.3)$ & 0.133 \\
\hline Heart failure, n (\%) & $29(5.6)$ & $18(18.0)$ & $11(2.6)$ & 0.000 \\
\hline CHADS2 & $1(0,2)$ & $1(1,2)$ & $1(0,2)$ & 0.119 \\
\hline CHADS-VASC & $2(1,3)$ & $2(1,4)$ & $2(1,3)$ & 0.219 \\
\hline Persistent AF, n(\%) & $158(30.4)$ & $72(72.0)$ & $86(20.5)$ & 0.000 \\
\hline $\begin{array}{l}\text { Left atrium diameter, } \\
\mathrm{mm}\end{array}$ & $41(36,45)$ & $46(44,49)$ & $39(36,43)$ & 0.000 \\
\hline $\begin{array}{l}\text { right atrium diameter, } \\
\mathrm{mm}\end{array}$ & $36(33,39)$ & $44(42,47)$ & $35(33,37)$ & 0.000 \\
\hline RVIDD, mm & $16(16,18)$ & $16(16,19)$ & $16(16,17)$ & 0.009 \\
\hline LVEF, \% & $66(60,70)$ & $63(55,68)$ & $66(61,70)$ & 0.000 \\
\hline LVIDD, mm & $50(47,54)$ & $53(49,56)$ & $50(47,53)$ & 0.000 \\
\hline EDV, & $122(104,140)$ & $135(112,155)$ & $119(103,135)$ & 0.000 \\
\hline Cardiac output, (L/s) & $5.5(4.7,6.6)$ & $5.95(5,7)$ & $5.4(4.7,6.4)$ & 0.008 \\
\hline $\operatorname{PVmax},(\mathrm{cm} / \mathrm{s})$ & $100(87,111)$ & $94.5(80,108)$ & $100(88,112)$ & 0.005 \\
\hline $\operatorname{AVmax},(\mathrm{cm} / \mathrm{s})$ & $133(117,152)$ & $125(110,141)$ & $135(118,154)$ & 0.002 \\
\hline Mitral E peek, $(\mathrm{cm} / \mathrm{s})$ & $88(70,107)$ & $100(82,116)$ & $85(69,106)$ & 0.000 \\
\hline Tricuspid E peek, (cm/s) & $56(49,64)$ & $59(51,69)$ & $55(49,63)$ & 0.011 \\
\hline Procedure time, $\mathrm{min}$ & $120(92.5,150)$ & $140(115,180)$ & $110(90,140)$ & 0.000 \\
\hline $\begin{array}{l}\text { PVI+linear ablation, } \mathrm{n} \\
(\%)\end{array}$ & $273(52.6)$ & $88(88.0)$ & $185(44.2)$ & 0.000 \\
\hline Cardioversion, $\mathrm{n}(\%)$ & $146(28.2)$ & $68(68.0)$ & $78(18.7)$ & 0.000 \\
\hline Recurrence, n (\%) & $170(32.8)$ & $52(52.0)$ & $118(28.2)$ & 0.000 \\
\hline Reablation, $\mathrm{n}(\%)$ & $117(22.5)$ & $33(33.0)$ & $84(20.0)$ & 0.007 \\
\hline
\end{tabular}

CAD: coronary artery disease. RVIDD: right ventricular internal diameter at end-diastole. LVEF: left ventricular ejection fraction. LVIDD: left ventricular internal diameter at end-diastole. EDV: end-diastole volume. PVmax: pulmonary artery maximum velocity. AVmax: aortic maximum velocity.

After an average of $33.11 \pm 21.45$ months, atrial arrhythmias recurred in 170 patients, and could be well controlled by anti-arrhythmic drugs in 53 patients. However, for those that were refractory to anti-arrhythmic drugs or those that could not bear the side effects of anti-arrhythmic drugs, reablation was applied in the rest 117 patients. The difference between two groups was listed in Table 3. As in Table 3 , the age was older, the CHADS-VASC scale was higher and the proportion of CAD was lower among the patients that recurrent AF could be controlled by drugs.

Table 3. Comparison between the two groups of patients with recurrence of $\mathrm{AF}$

\begin{tabular}{lllll}
\hline Value & Recur $(\mathrm{n}=170)$ & Reablation $(\mathrm{n}=117)$ & Drugs $(\mathrm{n}=53)$ & $\mathrm{P}$ \\
\hline Age, years & $65.39 \pm 10.65$ & $65.82 \pm 9.33$ & $69.49 \pm 8.49$ & 0.016 \\
Sex (female), $\mathrm{n}(\%)$ & $74(43.5)$ & $50(42.7)$ & $24(45.3)$ & 0.442 \\
Persistent AF, n(\%) & $76(44.7)$ & $47(40.2)$ & $24(54.7)$ & 0.055 \\
Hypertension, n (\%) & $98(57.6)$ & $70(59.8)$ & $28(52.8)$ & 0.245 \\
Diabetes mellitus, $\mathrm{n}(\%)$ & $35(20.6)$ & $20(17.1)$ & $15(28.3)$ & 0.073 \\
CAD, n(\%) & $35(20.6)$ & $19(16.2)$ & $16(30.2)$ & 0.032 \\
Heart failure, n (\%) & $11(6.5)$ & $6(5.1)$ & $5(9.4)$ & 0.231 \\
CHADS2 & $1(0,2)$ & $1(0,2)$ & $1(0.5,2)$ & 0.106 \\
CHADS-VASC & $2(1,3.75)$ & $2(1,3)$ & $2(1,3)$ & 0.016 \\
Left atrium diameter, mm & $42(39,46)$ & $42(39,46)$ & $44(39.5,46)$ & 0.566 \\
Right atrium diameter, mm & $37.5(34,42)$ & $37(34,41)$ & $39(35,44.5)$ & 0.141 \\
LVEF, \% & $65(59,70)$ & $65(59,70)$ & $65(58,69)$ & 0.976 \\
LVIDD, mm & $51(47,54)$ & $51(47,54)$ & $50(46,55)$ & 0.276 \\
Cardiac output, (L/s) & $5.5(4.8,6.6)$ & $5.4(4.9,6.6)$ & $5.7(4.5,6.6)$ & 0.784 \\
Mitral E peek, (cm/s) & $96.5(75,111)$ & $94(76,110)$ & $101(75,122)$ & 0.190 \\
\hline CAD: coronary artery disease. LVEF: left ventricular ejection fraction. LVIDD: left \\
ventricular internal diameter at end-diastole. & &
\end{tabular}

\section{Association of reablation with clinical characters.}

The correlation between reablation and persistent $\mathrm{AF}(\mathrm{r}=0.114, \mathrm{P}=0.009)$, left atrium diameter $(\mathrm{r}=0.150, \mathrm{P}=0.001)$, BAE $(\mathrm{r}=0.122, \mathrm{P}=0.005)$ were moderate. Reablation was also correlated with mitral $\mathrm{E}$ peek $(\mathrm{r}=0.092, \mathrm{P}=0.036)$ and right atrium diameter $(\mathrm{r}=0.092, \mathrm{P}=0.036$, as in Table 3$)$.

Receiver operating characteristic (ROC) curves were built to establish the values that represented the cutoff point of right atrium diameter (RAD) and left atrium diameter (LAD) with the greatest sensitivity and specificity to predict reablation (as Figure 2). A cutoff value of LAD $38.5 \mathrm{~mm}$ was associated with an area under the curve (AUC) of 0.603 and standard deviation of 0.029 (95\% CI 0.546-0.660, P = 0.001). The cutoff value of RAD $38.5 \mathrm{~mm}$ was associated with area under the curve (AUC) of 0.589 and standard deviation of 0.030 (95\% CI $0.531-0.648, \mathrm{P}=0.003)$.

Table 4. Correlations of reablation with clinical characters

\begin{tabular}{llllll}
\hline & $\mathrm{r}$ & $\mathrm{P}$ & & $\mathrm{r}$ & $\mathrm{P}$ \\
\hline Age & 0.21 & 0.626 & LAD & 0.150 & $0.001^{* *}$ \\
Sex & 0.27 & 0.538 & RAD & 0.092 & $0.036^{*}$ \\
Persistent AF & 0.114 & $0.009^{* *}$ & LVEF & -0.022 & 0.625 \\
Hypertension & 0.047 & 0.284 & LVIDD & 0.029 & -0.509 \\
Diabetes mellitus & -0.056 & 0.199 & EDV & 0.033 & 0.448 \\
CAD & -0.005 & 0.913 & ME & 0.092 & $0.036^{*}$ \\
Heart failure & -0.011 & 0.806 & TE & 0.034 & 0.439 \\
BAE & 0.122 & $0.005^{* *}$ & & &
\end{tabular}

CAD: coronary artery disease. HF: heart failure. LAD: left atrium diameter. RAD: right atrium diameter. LVEF: left ventricular ejection fraction. LVIDD: left ventricular internal diameter at end-diastole. EDV: end-diastole volume. ME: mitral E peak. TE: tricuspid E peak. BAE: biatrial enlargement.

** $\mathrm{P}<0.01$. * $\mathrm{P}<0.05$.

All the following confounders that potentially might have effect on procedural outcome based on prior knowledge or expected clinical relevance were entered into a Cox regression model. The variables were gender, age, persistent $\mathrm{AF}$, hypertension, diabetes, coronary artery disease, LVEF, EDV, BAE, Mitral E peak. Multivariate Cox regression analysis demonstrated that BAE (HR=1.755, 95\%CI 1.153-2.670, $\mathrm{P}=0.009$ ) was the only independent predictor for reablation, as in Table 5. Kaplan-Meier survival analysis curve showed significant difference of cum hazard of reablation between those with BAE and Non-BAE (log-rank P = 0.007, Figure 3).

\section{Subgroup analysis based on biatrial enlargement (BAE) in patients with left atrium enlargement.}

In order to determine the effects of left atrium diameter on the reablation, we carried out subgroup analysis based on BAE $(100 / 519)$ in patients with LAE $(287 / 519)$. We found that procedure time, left atrium 
diameter, LVEF, heart failure, recurrence, reablation were different significantly between LAE only and BAE patients. See Table 6.

Table 5. Multivariate Cox regression analysis of reablation

\begin{tabular}{llll}
\hline & HR & $95 \%$ C.I & $\mathrm{P}$ \\
\hline Age & 1.001 & $0.978-1.024$ & 0.942 \\
Sex & 1.067 & $0.702-1.622$ & 0.763 \\
Persistent AF & 0.746 & $0.474-1.174$ & 0.205 \\
HT & 1.055 & $0.681-1.636$ & 0.809 \\
DM & 1.371 & $0.790-2.377$ & 0.262 \\
CAD & 0.762 & $0.443-1.309$ & 0.325 \\
LVEF & 1.010 & $0.980-1.040$ & 0.525 \\
EDV & 0.998 & $0.991-1.005$ & 0.603 \\
Mitral E peek & 1.004 & $0.997-1.011$ & 0.288 \\
BAE & 1.755 & $1.153-2.670$ & 0.009 \\
\hline
\end{tabular}

Table 6. Subgroup analysis based on BAE in patients with LAE

\begin{tabular}{llll}
\hline Value & $\mathrm{BAE}(\mathrm{n}=100)$ & $\mathrm{LAE}$ only $(\mathrm{n}=187)$ & $\mathrm{P}$ \\
\hline Heart failure, $\mathrm{n}(\%)$ & $18(18)$ & $9(4.8)$ & 0.000 \\
Persistent AF, $\mathrm{n}(\%)$ & $72(72)$ & $68(36.4)$ & 0.000 \\
Left atrium diameter, mm & $46(44,49)$ & $43(41,46)$ & 0.000 \\
Right atrium diameter, mm & $44(42,47)$ & $37(35,38)$ & 0.000 \\
RVEDD, mm & $16(16,19)$ & $16(16,17)$ & 0.002 \\
LVEF, \% & $63(55,68)$ & $66(60,70)$ & 0.001 \\
LVEDD, mm & $53(49,56)$ & $52(48,54)$ & 0.121 \\
EDV, mL & $135(112,155)$ & $128(111,146)$ & 0.110 \\
Tricuspid E peek, (cm/s) & $59(51,69)$ & $55(48,63)$ & 0.018 \\
Procedure time, min & $140(115,180)$ & $120(100,150)$ & 0.001 \\
PVI+linear ablation, $\mathrm{n}(\%)$ & $88(88)$ & $105(56.1)$ & 0.000 \\
Electrical Cardioversion, $\mathrm{n}(\%)$ & $68(68)$ & $61(32.8)$ & 0.000 \\
Recurrence, $\mathrm{n}(\%)$ & $52(52)$ & $70(37.4)$ & 0.000 \\
Reablation, $\mathrm{n}(\%)$ & $33(33)$ & $49(26.2)$ & 0.001 \\
\hline
\end{tabular}

Multivariate Cox regression analysis demonstrated that BAE (HR 1.620, 95\% CI

$1.020-2.574, \mathrm{P}=0.041$ ) was an independent predictor for reablation among the

patients with LAE. See Table 7. And, BAE was also associated with more reablation

(Log rank $\mathrm{P}=0.026$ ) in these patients, see Figure 4
Table 7. Multivariate Cox regression analysis of reablation among the patients with LAE

\begin{tabular}{llll}
\hline & HR & $95 \%$ C.I & P \\
\hline Age & 1.006 & $0.977-1.036$ & 0.700 \\
Sex & 0.860 & $0.504-1.467$ & 0.579 \\
Persistent AF & 0.690 & $0.413-1.152$ & 0.156 \\
HT & 1.129 & $0.668-1.908$ & 0.650 \\
DM & 1.156 & $0.521-2.564$ & 0.722 \\
CAD & 0.785 & $0.396-1.555$ & 0.488 \\
LVEF & 0.991 & $0.961-1.023$ & 0.574 \\
EDV & 0.999 & $0.992-1.007$ & 0.876 \\
Mitral E peek & 1.003 & $0.994-1.011$ & 0.513 \\
BAE & 1.620 & $1.020-2.574$ & 0.041
\end{tabular}

\section{Discussion}

As we know, anti-arrhythmic drugs or cardioversion were used to control the recurred atrial arrhythmias for the patients with recurrence of $\mathrm{AF}$ after ablation. In our data, atrial arrhythmias recurred in 170 patients, and could be well controlled by anti-arrhythmic drugs in 53 patients. We compared the difference of the clinical characters between the patients with drugs and the patients underwent reablation to control the recurrent $\mathrm{AF}$, and found out that the patients with drugs were older, the CHADS-VASC scale of them was higher and the proportion of coronary artery disease was lower.

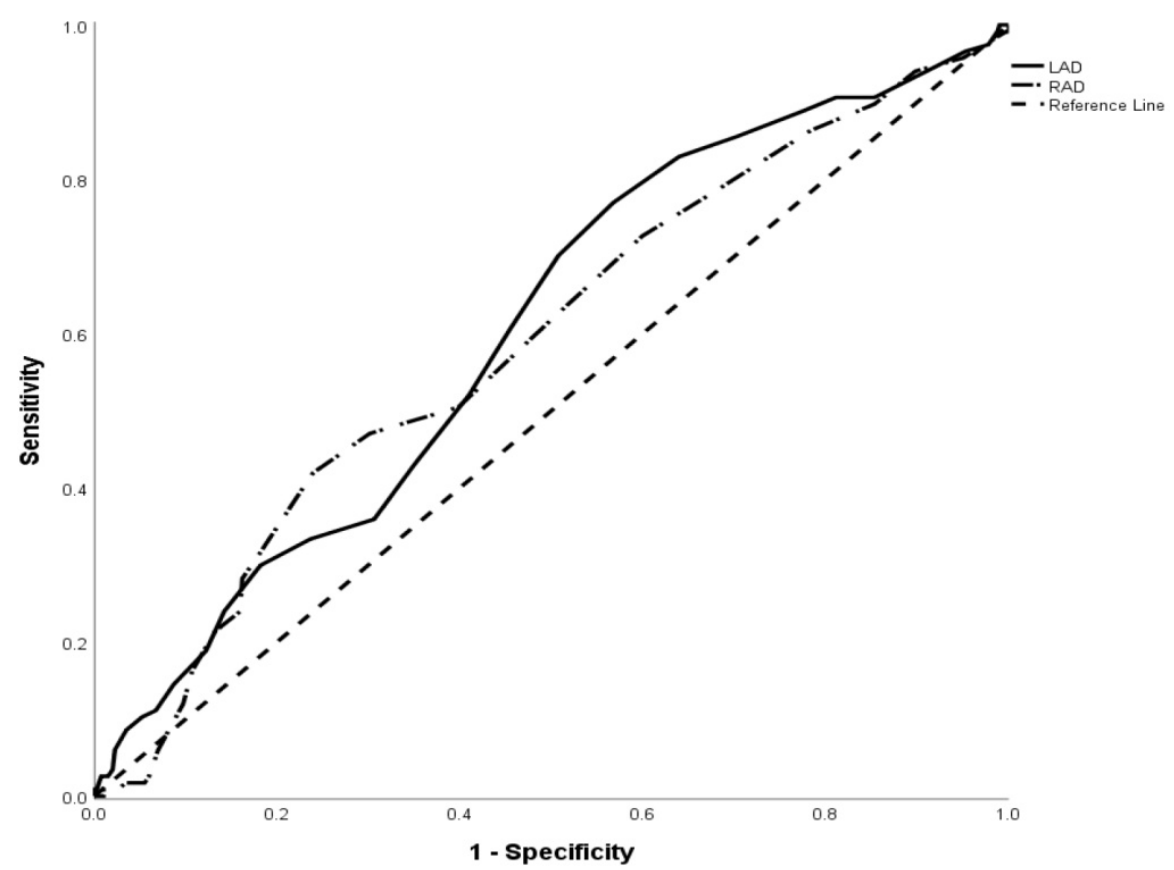

Figure 2. Receiver operating characteristic (ROC) curve showed the ability of RAD, $L A D$ in predicting reablation in atrial fibrillation patients. A cutoff value of $L A D 38.5 \mathrm{~mm}$ was associated with an area under the curve (AUC) of 0.603 and standard deviation of $0.029(95 \% \mathrm{Cl} 0.546-0.660, P=0.001)$. The cutoff value of RAD $38.5 \mathrm{~mm}$ was associated with area under the curve (AUC) of 0.589 and standard deviation of $0.030(95 \% \mathrm{Cl} 0.531-0.648, \mathrm{P}=0.003)$. 


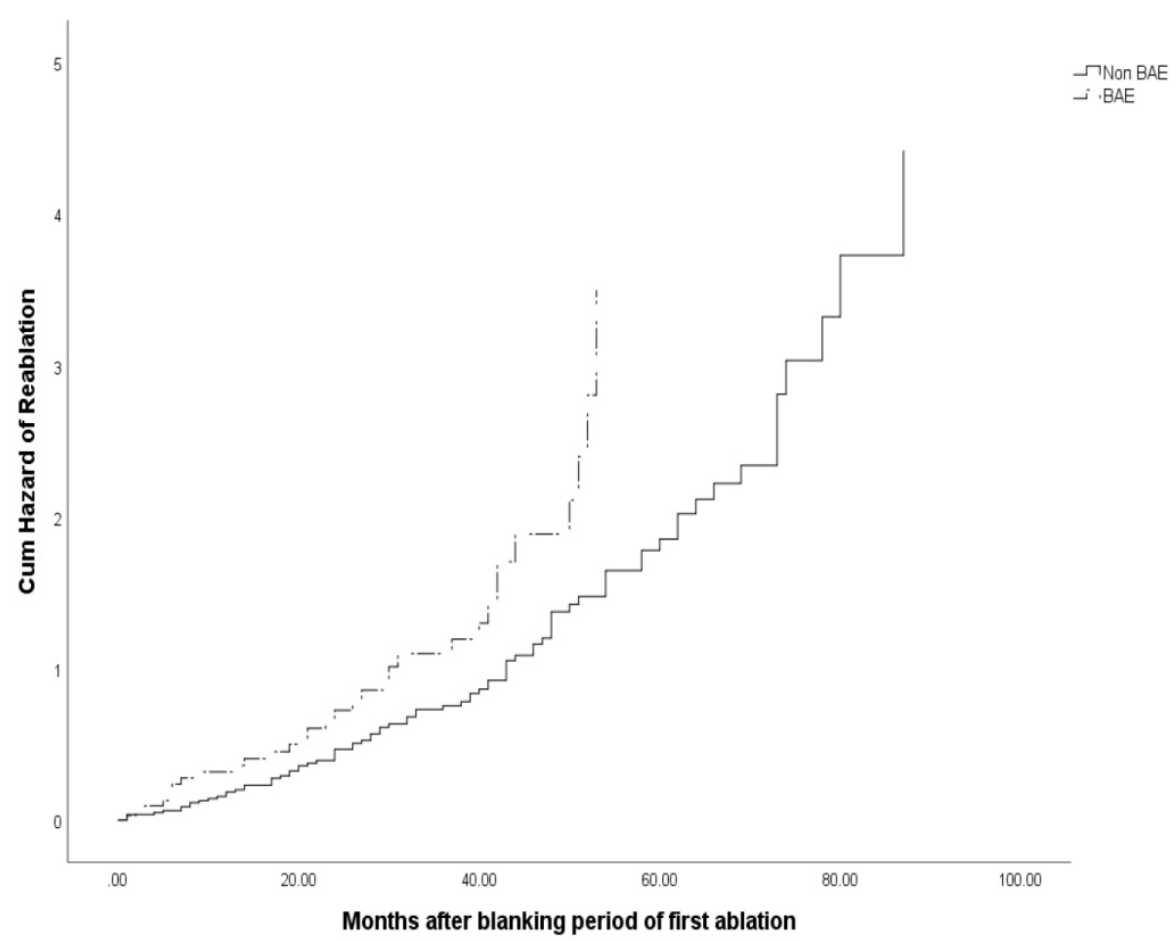

Figure 3. Cum hazard of reablation of patients with BAE or Non-BAE.

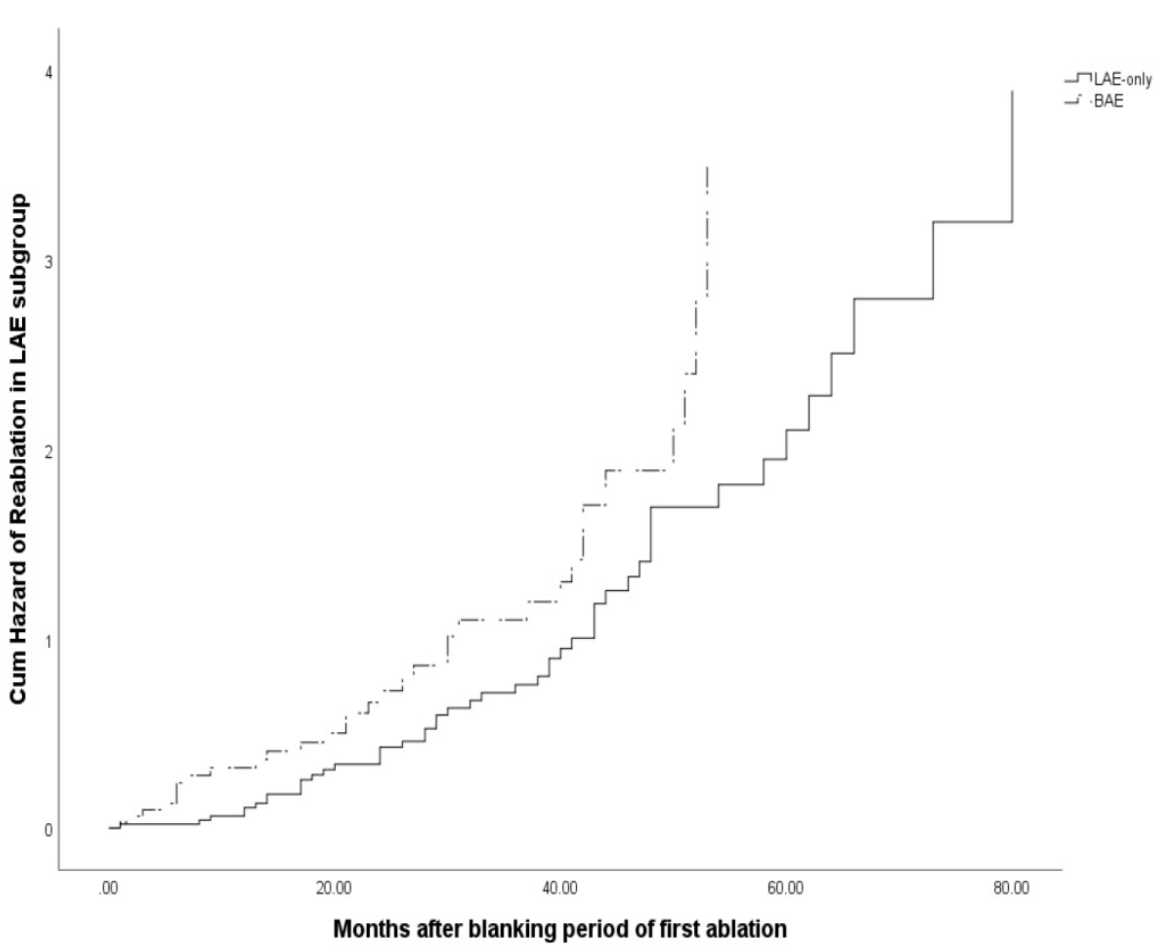

Figure 4. Cum hazard of reablation for subgroup analysis based on BAE in LAE group.

Recent studies $(15,16)$ had demonstrated that right atrium remodeling was associated with atrial fibrillation and right atrium structure and functions were closely associated with AF development. In our research, we found that the process duration was longer and the proportion of PVI plus additional lines was higher for patients with BAE. For BAE patients in our data, the proportion of persistent atrial fibrillation was higher and left atrium diameter was larger. Since left atrium remodeling was associated with atrial fibrillation and increased atrial volume, interstitial fibrosis, and increased myocardial stretch favor the sustainability of atrial fibrillation (17). Thus, we could suppose that atrial fibrosis in BAE patients might be more severe than that in Non-BAE patients. Maybe that was why longer process time and PVI plus 
additional lines were common for BAE patients in first ablation.

Left atrium size and right atrium diameter were found to be associated with recurrence of AF after catheter ablation $(7,8,9,18)$. In our study, there were 170 patients that recurred atrial arrhythmias, including paroxysmal $\mathrm{AF}$, atrial flutter, atiral tachycardia. Some of them could be controlled by anti-arrhythmic drugs, and for these patients, anticoagulation strategy was strictly carried out according to CHADS-VASC grading system. In the reablation, we found that ablation of pulmonary veins and lines in left atrium could terminate most of recurred atrial arrhythmias. Thus, gaps in pulmonary veins and incomplete block of left atrium ablation lines led to the recurrence of atrial arrhythmias in most patients in our data.

Although redo AF ablation was substantially more effective than $\mathrm{AAD}$ in reducing the progression and prevalence of $\mathrm{AF}$ after the failure of an initial ablation (19), it was not everyone who had recurred AF after catheter ablation that needed reablation, especially for those patients that atrial arrhythmia could be well controlled by anti-arrhythmic drugs. Recent multicenter clinical research in Europe had demonstrated that $9 \%$ of patients received a repeat ablation after PVI and $11 \%$ of patients were re-ablated after PVI plus additional lines or CFAE in left atrium. The proportion of the use of anti-arrhythmic drugs was decreased after first ablation and almost 32\%-34\% of patients used anti-arrhythmic drugs at 12 months follow up (6). In our data, almost 70\% (117/170) of patients with recurred atrial arrhythmia underwent reablation. The reason lay in that our follow up time was longer (33.11 \pm 21.45 months VS 12 months) and the constituent ratio of the patients were also different.

Receiver operating characteristic (ROC) curves was built to establish the values that represented the cutoff point of RAD and LAD to predict reablation. We found the cutoff values of LAD and RAD were both $38.5 \mathrm{~mm}$. According to TTE, LAE was diagnosed when LAD was above $40 \mathrm{~mm}$ for male and $35 \mathrm{~mm}$ for female, while RAE was diagnosed from that RAD was above $40 \mathrm{~mm}$ regardless of sex. From ROC curve, we knew that the sensitivity of prediction of reablation for LAD $35 \mathrm{~mm}$ (diagnose of LAE for female) was $88.9 \%$ and the specificity was $22.4 \%$. While for male, the sensitivity of the LAD $40 \mathrm{~mm}$ (diagnose of LAE for male) was $60.7 \%$ and the specificity was $54.5 \%$. However, the sensitivity of prediction of reablation for RAD 40mm (diagnose of RAE regardless of sex) was $28.2 \%$ and specificity was $83.8 \%$. Since the AUC was not so large (only 0.603 for LAD), the strength of prediction power for reablation for diameter of atrium was only moderate.

As left atrium enlargement was independent predictor for occurrence of $\mathrm{AF}$ after first or repeat ablation $(8,18)$, we did the subgroup analysis based on the BAE in LAE patients to evaluate the effects of left atrium diameter on the reablation. We found that left ventricular ejection fraction was decreased (BAE 63 $(55,68)$ VS LAE-only 66 $(60,70) \mathrm{P}=0.001)$, and heart failure were more common (BAE 18/100 VS LAE-only 9/187, $\mathrm{P}=0.000$ ), which meant that left ventricular systolic function was even worse for BAE patients. Also, persistent atrial fibrillation, additional lines ablation besides PVI and electrical cardioversion were more common among these patients in the first ablation process. Multivariate Cox regression analysis showed that BAE (HR 1.620, 95\% CI 1.020-2.574, $\mathrm{P}=0.041$ ) was an independent predictor for reablation among the patients with enlarged left atrium. Kaplan-Meier survival analysis demonstrated that BAE was also associated with more reablation (Log rank $\mathrm{P}=0.026$ ) in these patients.

\section{Limitations}

This was retrospective study and was only a single institutional study. For criteria of reablation, we had to take the patients' desire into consideration, thus subjective factors would affect the result. In our data, reporting and selection bias could not be fully excluded. All comparisons between groups have to be interpreted with caution because PVI plus additional lines may have been performed more frequently in patients with more extensive substrate who would have had an even poorer prognosis with a PVI approach. Due to the non-standardized arrhythmia screening success rates may be overestimated.

\section{Conclusions}

Biatrial enlargement (BAE) is an independent predictor for reablation in atrial fibrillation patients and is associated with reablation in our follow up.

\section{Competing Interests}

The authors have declared that no competing interest exists.

\section{References}

1. Lane DA, Skjøth F, Lip GYH, Larsen TB, Kotecha D. Temporal trends in incidence, prevalence, and mortality of atrial fibrillation in primary care. J Am Heart Assoc 2017; 6: e005155.

2. Haïssaguerre M, Jaïs P, Shah DC, Takahashi A, Hocini M, Quiniou G, et al. Spontaneous initiation of atrial fibrillation by ectopic beats originating in the pulmonary veins. NEngl JMed. 1998; 339: 659-66.

3. Wilber DJ, Pappone C, Neuzil P, De Paola A, Marchlinski F, Natale A et al. Comparison of antiarrhythmic drug therapy and radiofrequency catheter ablation in patients with paroxysmal atrial fibrillation: a randomized controlled trial. JAMA 2010; 303: 333-40.

4. Morillo CA, Verma A, Connolly SJ, Kuck KH, Nair GM, Champagne J et al. Radiofrequency ablation vs antiarrhythmic drugs as first-line treatment of paroxysmal atrial fibrillation (RAAFT-2): a randomized trial. JAMA 2014; 311: 692-700. 
5. Cappato R, Calkins H, Chen SA, Davies W, Iesaka Y, Kalman J et al. Worldwide survey on the methods, efficacy, and safety of catheter ablation for human atrial fibrillation. Circulation 2005; 111: 1100-5.3.

6. Boris Schmidt, Josep Brugada, Elena Arbelo, et al. Ablation strategies for different types of atrial fibrillation in Europe: results of the ESC-EORP EHRA Atrial Fibrillation Ablation Long-Term registry. Europace. 2020; 22: 558-566.

7. Jian-hui Zhang, Yi Wang, Kai Tang, et al. Association between left atrial size and atrial fibrillation recurrence after single circumferential pulmonary vein isolation: a systematic review and meta-analysis of observational studies. Europace 2012;14:638-645.

8. Ri-Bo Tang, Xian-Liang Yan, Jian-Zeng Dong, et al. Predictors of recurrence after a repeat ablation procedure for paroxysmal atrial fibrillation: role of left atrial enlargement. Europace 2014; 16: 1569-1574.

9. Song-Nan Wen, Nian Liu, Rong Bai, Ri-Bo Tang, Rong-Hui Yu, De-Yong Long, Cai-Hua Sang, et al. Right atrial diameter and outcome of catheter ablation of atrial fibrillation. J Interv Card Electrophysiol 2017; 49(2): 157-164.

10. Marrouche NF, Wilber D, Hindricks G, et al. Association of atrial tissue fibrosis identified by delayed enhancement MRI and atrial fibrillation catheter ablation: The DECAAF study. JAMA 2014; 311: 498-506.

11. Konstantinos Vlachos, Michael Efremidis, Konstantinos P.Letsas, et al. Low-voltage areas detected by high-density electroanatomical mapping predict recurrence after ablation for paroxysmal atrial fibrillation. J Cardiovasc electrophysiol 2017; 28: 1393-1402

12. Yu Kumagai, Tadateru Iwayama, Takanori Arimoto, et al. Biatrial volume, estimated using magnetic resonance imaging, predicts atrial fibrillation recurrence after ablation. Pacing Clin Electrophysiol 2018; 41: 1635-1642.

13. The consensus statements endorsed by the Beijing Task Force on echocardiography. [Standard techniques and normal value of echocardiography measurements in the Chinese population]. 1st ed. Beijing, Scientific and Technical Documentation Press; 2005.

14. Dong JZ, Sang CH, Yu RH, Long DY, Tang RB, Jiang CX, et al. Prospective randomized comparison between a fixed ' $2 \mathrm{C} 3 \mathrm{~L}$ ' approach vs stepwise approach for catheter ablation of persistent atrial fibrillation. Europace. 2015; 17: 1798-806.

15. Pyankov V, Chuyasova $Y$, Yatka V, et al. Right atrium remodeling in patients with arterial hypertension and atria fibrillation. Journal of Hypertension 2010; 28, e-Supplement A, pp.1.47.

16. Ugur Aksu, Kamuran Kallan, Oktay Gulcu, et al. The role of the right atrium in development of postoperative atrial fibrillation: A speckle tracking echocardiography study. J Clin Ultrasound. 2019; 47: 470-476.

17. Riccardo M. Inciardi and Andrea Rossi. Left atrium: a forgotten biomarker and a potential target in cardiovascular medicine. J Cardiovasc Med 2019; 12: 797-808

18. Zhuang J, Wang Y, Tang K, et al. Association between left atrial size and atrial fibrillation recurrence after single circumferential pulmonary vein isolation: a systematic review and meta-analysis of observational studies. Europace 2012; 14: 638-45.

19. Evgeny P, Alexander R, Mirko DM, et al. Progression of atrial fibrillation after a failed initial ablation procedure in patients with paroxysmal atrial fibrillation. Circ Arrhythm Electrophysiol 2013; 6: 754-760 